\title{
WEIGHTED LIAPUNOV FUNCTIONS FOR A CLASS OF THIRD-ORDER AUTONOMOUS DIFFERENTIAL EQUATIONS*
}

BY

\author{
LARRY R. ANDERSON
}

Whitman College, Walla Walla, Washington

1. Introduction. In 1963, Walter Leighton published a paper [7] in which he provided Liapunov functions for general classes of second- and third-order differential equations. In a subsequent paper [3], the current author and Walter Leighton considered Liapunov functions for second-order systems that were more general than those given in Leighton's 1963 paper. Further, this latter paper gave a class of weighted Liapunov functions for certain second-order systems. Further work utilizing weighted Liapunov functions for second-order equations was published by the current author in [1] and by S. Duchich and the current author in [2]. A separate paper by A. Skidmore [8] extended Leighton's 1963 work on third-order equations to fourth-order equations, but no "weighting" was considered in either paper.

The purpose of this paper is to extend Leighton's work (in [7]) on third-order systems by providing a class of weighted Liapunov functions for an equation

$$
\dddot{x}+\phi(x, \dot{x}, \ddot{x})=0
$$

and the associated system

$$
\dot{x}=y, \quad \dot{y}=z, \quad \dot{z}=-\phi(x, y, z) .
$$

This system is a bit more general than third-order systems considered by Leighton in [7]. Moreover, for third-order systems considered by Leighton in [7], our weight functions $V_{w}(w=$ weight $)$ reduce to those given by Leighton when $w=1$. In turn, as pointed out by Leighton in [7], his Liapunov functions are "sharp" in that they provide stability criteria in linear cases when the Routh-Hurwitz condition holds. Thus our class of functions provides breadth and sensitivity.

* Received June 4, 1986. 
2. A class of Liapunov functions. We assume here that $\phi$ is of class $C^{\prime \prime}$ in an open set containing the origin and that the origin is an isolated critical point of (1.1).

We will consider weight functions $w(x, y, z)$ which are of class $C^{\prime \prime}$ neighboring the origin and are positive in a deleted neighborhood of the origin.

For such a function $w$ and a constant $\alpha$, we define

$$
\begin{aligned}
V_{w}(x, y, z)= & \int_{0}^{z} v w(x, 0, v) d v+\int_{0}^{y} w(x, u, 0) \phi(x, u, 0) d u \\
& +\alpha\left\{z y w(x, y, 0)+\int_{0}^{x} w(t, 0,0) \phi(t, 0,0) d t\right. \\
& \left.+\int_{0}^{y} u\left[w(x, u, 0) \phi_{z}(x, u, 0)+w_{z}(x, u, 0) \phi(x, u, 0)\right] d u\right\} .
\end{aligned}
$$

If $w \equiv 1$ and if $\phi(x, y, z)=z \psi(x, y)+\theta(x, y)$, then this reduces to the function considered by Leighton in [7].

In the sequel it will be convenient to define

$$
A(x, y)=w(x, y, 0) \phi(x, y, 0)
$$

and

$$
B(x, y)=y w(x, y, 0) \phi_{z}(x, y, 0)+y w_{z}(x, y, 0) \phi(x, y, 0) .
$$

To compute $\dot{V}_{w}$ we first compute the various first partial derivatives. We obtain

$$
\begin{aligned}
\frac{\partial V_{w}}{\partial x}= & \int_{0}^{z} v w_{x}(x, 0, v) d v+\int_{0}^{y}\left(A_{x}(x, u)+\alpha B_{x}(x, u)\right) d u \\
& +\alpha w(x, 0,0) \theta(x, 0,0)+\alpha z y w_{x}(x, y, 0), \\
\frac{\partial V_{w}}{\partial y}= & A(x, y)+\alpha w(x, y, 0) z+\alpha z y w_{y}(x, y, 0)+\alpha B(x, y), \\
\frac{\partial V_{w}}{\partial z}= & z w(x, 0, z)+\alpha y w(x, y, 0) .
\end{aligned}
$$

We then have

$$
\begin{aligned}
\dot{V}_{w}= & y \int_{0}^{z} v w_{x}(x, 0, v) d v+y \int_{0}^{y}\left(A_{x}(x, u)+\alpha B_{x}(x, u)\right) d u \\
& +\alpha y w(x, 0,0) \phi(x, 0,0)+\alpha z y^{2} w_{x}(x, y, 0) \\
& +z\left[w(x, y, 0) \phi(x, y, 0)+\alpha w(x, y, 0) z+\alpha z y w_{y}(x, y, 0)+\alpha B(x, y)\right] \\
& -\phi(x, y, z)[z w(x, 0, z)+\alpha y w(x, y, 0)] \\
= & y^{2}\left[\frac{1}{y} \int_{0}^{y}\left(A_{x}(x, u)+\alpha B_{x}(x, u)\right) d u\right]+z^{2}\left[\alpha w(x, y, 0)+\alpha y w_{y}(x, y, 0)\right] \\
& +z y\left(\frac{\alpha B(x, y)}{y}+\frac{1}{z} \int_{0}^{z} v w_{x}(x, 0, v) d v+\alpha y w_{x}(x, y, 0)\right) \\
& +z w(x, y, 0) \phi(x, y, 0)+\alpha y w(x, 0,0) \phi(x, 0,0) \\
& -z \phi(x, y, z) w(x, 0, z)-\alpha y w(x, y, 0) \phi(x, y, z) \\
= & C y^{2}+D z y+E z^{2},
\end{aligned}
$$


where $C, D$, and $E$ are given by

$$
\begin{aligned}
C= & \frac{1}{y} \int_{0}^{y}\left(A_{x}(x, u)+\alpha B_{x}(x, u)-A_{y}(x, u)\right) d u \\
D= & \frac{\alpha B(x, y)}{y}+\frac{1}{z} \int_{0}^{z}\left(z w_{x}(x, 0, v)-\alpha w(x, y, 0) \phi_{z}(x, y, v)\right) d v \\
& +\alpha y w_{x}(x, y, 0)+\phi(x, y, z) \frac{1}{y} \int_{0}^{y} w_{y}(x, u, v) d u, \\
E= & \alpha w(x, y, 0)+\alpha y w_{y}(x, y, 0)-\frac{1}{z} \int_{0}^{z} \frac{\partial(\phi w)}{\partial z}(x, y, v) d v .
\end{aligned}
$$

We obtain the following result.

THEOREM 1. If there exists a weight function $w$ and a constant $\alpha$ such that $V_{w}$ is positive definite neighboring the origin and such that $E<0$ and $D^{2}-4 E C<0$, then the origin is an asymptotically stable critical point of (1.1) and $V_{w}$ is a Liapunov function for (1.1).

Here we assume that $\phi$ and $w$ satisfy the regularity conditions given at the beginning of Sec. 2 . Further, the theorem allows us to conclude asymptotic stability since the set $\dot{V}_{w}=0$ is the $x$ axis, which is an invariant set (see [4]).

Example 1. We let $w=1$ and find sufficient conditions for the asymptotic stability of the origin. If $\phi(x, y, z)=z \psi(x, y)+\theta(x, y)$, these conditions agree with those given in [7].

We first find conditions which insure that $V_{w}(w=1)$ will be locally positive definite. Consider the matrix

$$
\left(a_{i j}\right)=\left(\frac{\partial^{2} V_{w}}{\partial x_{i} \partial x_{j}}\right), \quad 1 \leqslant i \leqslant 3, \quad 1 \leqslant j \leqslant 3 .
$$

It is easily shown that

$$
\operatorname{det}\left(a_{i j}\right)^{0}=\left|\begin{array}{ccc}
\alpha \phi_{x}^{0} & \phi_{x}^{0} & 0 \\
\phi_{x}^{0} & \phi_{y}^{0}+\alpha \phi_{z}^{0} & \alpha \\
0 & \alpha & 1
\end{array}\right|,
$$

where the superscript denotes evaluation at the origin. If we assume that

$$
\phi_{z}^{0}>\alpha, \quad \phi_{x}^{0}>0, \quad \phi_{y}^{0}>0
$$

and let $\alpha=\left(\phi_{x}^{0}\right)\left(\phi_{y}^{0}\right)^{-1}$, then the principal minors of the above determinant will be positive and hence $V$ will be locally positive definite.

The discriminant $D^{2}-4 E C$ of $(2.1)$ evaluated at the origin is given by

$$
\left(D^{2}-4 E C\right)^{0}=(-4 E C)^{0}=-4\left(\alpha-\phi_{z}^{0}\right)\left(\phi_{x}^{0}-\phi_{y}^{0}\right),
$$

which will be negative neighboring the origin if $\phi_{x}^{0}<\phi_{y}^{0}$. Since $E^{0}=\alpha-\phi_{z}^{0}$, it follows that $V_{w}(w=1)$ is a Liapunov function for (1.1) if $(2.2)$ holds and if $\phi_{x}^{0}<\phi_{y}^{0}$ (here $\left.\alpha=\left(\phi_{x}^{0}\right)\left(\phi_{y}^{0}\right)^{-1}\right)$. 
If the above conditions hold and $\phi$ contains higher-order terms in $z, V_{w}(w=1)$ is a Liapunov function. For instance, $V_{w}$ is a Liapunov function when $\phi(x, y, z)=4 z+z^{3}+$ $x+2 y$. Such cases weren't considered by Leighton in [7].

Now assume that $w$ is any weight function satisfying the regularity conditions given at the beginning of Sec. 2. Further, assume that $w^{0}>0$ and that (2.2) holds and that $\phi_{x}^{0}<\phi_{v}^{0}$. It can be shown that

$$
\operatorname{det}\left(\frac{\partial^{2} V_{w}^{0}}{\partial x_{i} \partial x_{j}}\right)=\left|\begin{array}{ccc}
\alpha w^{0} \phi_{x}^{0} & w^{0} \phi_{x}^{0} & 0 \\
w^{0} \phi_{x}^{0} & w^{0} \phi_{y}^{0}+\alpha w^{0} \phi_{z}^{0} & \alpha w^{0} \\
0 & \alpha w^{0} & w^{0}
\end{array}\right| .
$$

Also,

$$
\left(D^{2}-4 E C\right)^{0}=-4\left(\alpha w^{0}-w^{0} \phi_{z}^{0}\right)\left(w^{0} \phi_{x}^{0}-w^{0} \phi_{y}^{0}\right) .
$$

We obtain the following result, which in certain cases provides a large class of Liapunov functions for (1.1).

Theorem 2. Let $w_{0}>0$ and assume that $\phi$ satisfies (2.2) and that $\phi_{x}^{0}<\phi_{y}^{0}$. Then $V_{w}$ is a Liapunov function for the system (1.1), where $\alpha=\left(\phi_{x}^{0}\right)\left(\phi_{y}^{0}\right)^{-1}$.

In some earlier papers (see [1], [2], [3]) concerning second-order systems, particular attention was given to considering estimates of regions of asymptotic stability obtained from $V_{w}$ by varying the weight function $w$. For certain weight functions and certain second-order systems, one obtains better estimates than with the single estimate provided by $V_{w}$ with $w=1$. In certain cases (see [1], [2]) optimal estimates over certain subclasses of weight functions may be found.

The following example illustrates that some of these methods carry over to the third-order case.

Example 2. In the following example we consider a system (see [5, p. 228])

$$
\dot{x}=y, \quad \dot{y}=z, \quad \dot{z}=-y-b z-f(x) .
$$

Under appropriate conditions, the equation $V=V\left(P_{0}\right)$ will be an estimate of the region of asymptotic stability, where $V$ is the function above with $w=1$ for the system (2.3) and $V\left(P_{0}\right)$ is the smallest positive critical value of $V$. We will show how to choose a weight function $w$ such that the manifold $V_{w}=V_{w}\left(P_{0}\right)$ bounds a subregion of the region of asymptotic stability of the origin and wholly contains the region $V=V\left(P_{0}\right)$ (see, in particular, [2]).

We assume that $f \in C^{\prime}(-\infty, \infty)$, and that $f$ vanishes only at $x=0$ and at some $x_{0}>0$. We assume further that $0 \leqslant f^{\prime}(x)<m<1<b$ for all $x$, that $x f(x) \geqslant 0$ and $f^{\prime}(0)>0$, and that

$$
\lim _{x \rightarrow \infty} f(x)=L_{1} \quad \text { and } \quad \lim _{x \rightarrow-\infty} f(x)=L_{2},
$$

where $L_{1}$ and $L_{2}$ are finite. 
With $w=1$ and $\alpha=1$ we have

$$
V=\frac{z^{2}}{2}+\frac{y^{2}}{2}+f(x) y+z y+\int_{0}^{x} f(u) d u+\frac{b y^{2}}{2}
$$

and with $w=1+B x^{2}$ we have

$$
\begin{aligned}
V_{w}= & \frac{z^{2}}{2}\left(1+B x^{2}\right)+\frac{y^{2}}{2}\left(1+B x^{2}\right)+\left(1+B x^{2}\right) f(x) y \\
& +z y\left(1+B x^{2}\right)+\int_{0}^{x}\left(1+B u^{2}\right) f(u) d u+\frac{b y^{2}}{2}\left(1+B x^{2}\right) .
\end{aligned}
$$

We summarize several facts concerning $V$ and $V_{w}$ and omit the computational details.

(i) $V$ and $V_{w}$ are locally positive definite.

(ii) For $B$ sufficiently small and positive and for $B=0$, the points $\left(x_{0}, 0,0\right)$ and $(0,0,0)$ are the only finite critical points of $V_{w}$.

(iii) For all $B \geqslant 0, V_{w}$ has no infinite critical points, which follows from the fact that there is no sequence $\left\{X_{n}\right\}$ in $R^{3}$ such that

$$
\left|X_{n}\right| \rightarrow \infty \quad \text { and } \quad \frac{\partial V_{w}}{\partial x_{i}}\left(X_{n}\right) \rightarrow 0 \quad \text { as } n \rightarrow \infty(1 \leqslant i \leqslant 3) .
$$

(iv) From the above and [6], it follows that for $B$ small and positive

$$
V(x, y, z)=V\left(x_{0}, 0,0\right)
$$

and

$$
V_{w}(x, y, z)=V_{w}\left(x_{0}, 0,0\right)
$$

form closed 2 manifolds containing the origin, both of which lie in a subset of the region of asymptotic stability of the origin.

We now show that the region (2.5) wholly contains (2.4) and that for $B$ sufficiently small and positive, $\dot{V}_{w} \leqslant 0$ inside (2.5).

We first verify the latter. It may be shown that

$$
\begin{aligned}
\dot{V}_{w}= & z^{2}\left(x y B+\left(1+B x^{2}\right)(1-b)\right) \\
& +y^{2}\left(x y B+2 x f(x) B+2 x z B+x y b B+\left[f^{\prime}(x)-1\right]\left[1+B x^{2}\right]\right) .
\end{aligned}
$$

Since the region (2.5) is compact, it follows that for $B$ sufficiently small, $\dot{V}_{w} \leqslant 0$ inside this region.

To verify that (2.5) contains (2.4), we solve for $z$ in terms of $x$ and $y$. For the surface (2.5), we have that

$$
z=-y \pm 2^{1 / 2}\left[\frac{\int_{0}^{x_{0}}\left(1+B u^{2}\right) f(u) d u}{1+B x^{2}}-\frac{y^{2} b}{2}-f(x) y-\frac{\int_{0}^{x}\left(1+B u^{2}\right) f(u) d u}{1+B x^{2}}\right]^{1 / 2}
$$


and for (2.4) we have that

$$
\begin{aligned}
z & =-y \pm 2^{1 / 2}\left[\int_{0}^{x_{0}} f(u) d u-\int_{0}^{x} f(u) d u-\frac{b y^{2}}{2}-f(x) y\right]^{1 / 2} \\
& =-y \pm 2^{1 / 2} b^{-1 / 2}\left[\int_{0}^{x_{0}} f(u)\left[b-f^{\prime}(u)\right] d u-\frac{b^{2}}{2}\left(y+\frac{f(x)}{b}\right)^{2}\right. \\
& \left.-\int_{0}^{x} f(u)\left[b-f^{\prime}(u)\right] d u\right]^{1 / 2} .
\end{aligned}
$$

If we set the quantity in square brackets in (2.7) equal to zero, it may be seen that this is a closed curve bounding the domain of $z$ in (2.7). The projection of this curve on the $x$ axis is the interval $\left[x_{1}, x_{0}\right]$, where $x_{1}$ is the unique negative value such that

$$
\int_{0}^{x_{0}} f(u)\left(b-f^{\prime}(u)\right) d u=\int_{0}^{x_{1}} f(u)\left(b-f^{\prime}(u)\right) d u .
$$

To prove our assertion, it suffices to prove that the quantity in square brackets in (2.6) exceeds the quantity in square brackets in (2.7). This reduces to proving that

$$
\int_{x}^{x_{0}} u^{2} f(u) d u \geqslant x^{2} \int_{x}^{x_{0}} f(u) d u, \quad x_{1} \leqslant x \leqslant x_{0} .
$$

If we put

$$
G(x)=\int_{x}^{x_{0}} u^{2} f(u) d u-x^{2} \int_{x}^{x_{0}} f(u) d u
$$

and note that

$$
G^{\prime}(x)=-2 x \int_{x}^{x_{0}} f(u) d u, \quad x_{1} \leqslant x \leqslant x_{0},
$$

the inequality (which is strict in $\left.\left(x_{1}, x_{0}\right)\right)$ follows at once.

\section{REFERENCES}

[1] L. Anderson, Estimates of planar regions of asymptotic stability, Quart. Appl. Math. 36, 434-438 (1979)

[2] L. Anderson and S. Duchich, Weight functions for a class of Liapunov functions in the plane, Quart. Appl. Math. 38, 497-504 (1981)

[3] L. Anderson and W. Leighton, Liapunov functions for autonomous systems of second order, J. Math. Anal. Appl. 23, 645-664 (1968)

[4] J. P. LaSalle and S. Lefschetz, Stability by Liapunov's direct method, with applications, Academic Press, New York, 1961

[5] W. Leighton, An introduction to the theory of ordinary differential equations, Wadsworth, Belmont, Calif., 1976

[6] W. Leighton, Morse theory and Liapunov functions, Rend. Circ. Mat. Palermo (2), 1-10 (1964)

[7] W. Leighton, On the construction of Liapunov functions for certain autonomous nonlinear differential equations, Contrib. Differential Equations 2, 367-383 (1963)

[8] A. Skidmore, On the stability of solutions of a differential equation of fourth order, J. London Math. Soc. 41, 649-661 (1966) 